flow cytometry, in relation to clinical parameters and previously established LN classes assessed according to the ISN/RPS 2003 classification.

Results Lymphocytes percentages in class IV were different from classes III, V or a combination of III and V. In the latter classes, the percentages of the Tregs and Th17 cells were significantly lower, whereas in class IV the increase in FOXP3 in the Tregs and Th17 cells over six months period was significantly higher (Table 1). Changes in glomerular filtration rate and SLEDAI within 5 years did not correlate with single or repeated Tregs measurements.

Conclusions Differences in lymphocyte proportions between class IV and other classes may suggest its distinct pathogenesis and warrants further investigations on their role as $\mathrm{LN}$ biomarker.

\section{CLINICAL SIGNIFICANCE OF ANTI-DNA/NR2 ANTIBODIES IN DE NOVO NPSLE AND POST-STEROID NPSLE}

${ }^{1} \mathrm{Y}$ Fujieda*, ${ }^{2} \mathrm{~S}$ Mader, ${ }^{2} \mathrm{~V}$ Jegnathan, ${ }^{2} \mathrm{Y}$ Arimuna, ${ }^{1} \mathrm{Y}$ Shimizu, ${ }^{1} \mathrm{M}$ Kato, ${ }^{1} \mathrm{~K}$ Oku, ${ }^{1} \mathrm{~T}$ Bohgaki, ${ }^{1} \mathrm{O}$ Amengual, ${ }^{1} \mathrm{~S}$ Yasuda, ${ }^{2} \mathrm{~B}$ Diamond, ${ }^{1} \mathrm{~T}$ Atsumi. ${ }^{1}$ Hokkaido University Graduate School of Medicine, Division of Rheumatology- Endocrinology and Nephrology, Sapporo, Japan; ${ }^{2}$ The Feinstein Institute for Medical Research, Centre of Autoimmune and Musculoskeltal Diseases, New York, USA

10.1136/lupus-2017-000215.272

Background and aims Anti-DNA/NR2 antibodies are a subset of anti DNA autoantibodies that cross-react with the extracellular domain of the GluN2A/GluN2B subunits of the $\mathrm{N}$ methyl-d-aspartate receptor 2 (NR2), which induce apoptosis of hippocampus neurons and psychiatric disorder in mice and humans. Neuropsychiatric SLE (NPSLE) can develop after initiation of steroid (post-steroid neuropsychiatric manifestation: PSNP) or before treatment (de novo NPSLE). The objective of this study was to clarify the prevalence of anti-DNA/NR2 antibodies in PSNP-SLE and de novo NPSLE

Methods This study involved a cohort of patients with NPSLE who were admitted to Hokkaido University Hospital. NPSLE patients were classified into two groups, de novo NPSLE and PSNP-SLE. Serum anti-DNA antibodies and anti-DNA/NR2 antibodies were measured using in-house ELISAs.

Results Serum samples were obtained from 29 patients with de novo NPSLE, 26 with PSNP-SLE and 83 healthy controls (HC). The levels of anti-DNA antibodies in patients with de novo NPSLE and PSNP-SLE were significantly higher than those in healthy controls (de novo NPSLE, PSNP-SLE, HC: $1.34 \pm 0.09,1.40 \pm 0.14,0.33 \pm 0.03, \mathrm{p}<0.0001)$. The levels of anti-DNA/NR2 antibodies were highest in de novo NPSLE and in PSNP-SLE and HC (de novo NPSLE, PSNP-SLE, HC: 0.75 $\pm 0.10,0.60 \pm 0.07,0.49 \pm 0.03)$. In PSNP-SLE, the frequency of mood disorders was higher than that in de novo NPSLE (58\% vs $31 \% \mathrm{p}<0.05)$.

Conclusions The levels of anti-DNA/NR2 in PSNP-SLE are lower than in de novo NPSLE, indicating the differences in the pathogenesis of these two conditions.

\section{3 URINARY TWEAK LEVELS AS BIOMARKER OF LUPUS NEPHRITIS IN COLOMBIAN SLE PATIENTS}

'BL Ortiz Reyes, ${ }^{1} \mathrm{~T}$ Urrego, ${ }^{2,3} \mathrm{AL}$ Vanegas, ${ }^{4} \mathrm{G}$ Aroca, ${ }^{4} \mathrm{~A}$ Cadena Bonfanti, ${ }^{4} \mathrm{~L}$ Almendrales, ${ }^{2,3} \mathrm{CH}$ Muñoz, ${ }^{2} \mathrm{LA}$ González, ${ }^{4,5} \mathrm{~A}$ Iglesias, ${ }^{1,2} \mathrm{G}$ Vásquez, ${ }^{1,2} \mathrm{JA}$ Gómez-Puerta*. ${ }^{1}$ Universidad de Antioquia, Grupo de Inmunología Celular e Inmunogenética GICIC, Medellín, Colombia; ${ }^{2}$ Universidad de Antioquia, Grupo de Reumatología, Medellin, Colombia; ${ }^{3}$ Hospital San Vicente Fundación, Rheumatology, Medellin, Colombia; ${ }^{4}$ Universidad Simón Bolivar, Grupo investigación Nefrología, Barranquilla, Colombia; ${ }^{5}$ Universidad Nacional de Colombia, Facultad de Medicina, Bogotá, Colombia

\subsection{6/lupus-2017-000215.273}

Background and aims TNF-like WEAK inducer of apoptosis (TWEAK), a TNF ligand superfamily is mainly produced by monocytes/macrophages, and is widely expressed at the RNA level in tissues including kidneys. The usefulness of urinary TWEAK (uTWEAK) to identify renal involvement in Mestizo and African-Latin American (ALA) SLE patients has not been examined yet.

Methods Patients meeting the revised ACR criteria for SLE were recruited from 2 different centres at Medellín and Baranquilla, Colombia. uTWEAK were measured using an ELISA kit ( $\mathrm{R}$ and $\mathrm{D}$ system, USA)

Results 158 SLE patients were recruited (89\% female) with median age of $32.8 \pm 12.1$ years and median disease duration of 7.27 \pm 6.6 years. Mestizo (77\%) and ALA (20\%) were majority. $64 \%$ of patients had lupus nephritis (LN). 50 out of 71 biopsy proven LN had proliferative forms. Mean SLEDAI score was $8.5 \pm 8.7$. LN patients $(2803 \pm 6086$ vs $672 \pm 1042$, $\mathrm{p}=0.013) \quad($ Fig 1A) and ALA patients (3995 \pm 9656 vs 1618 $\pm 2653, p=0.002$ ) had significant higher levels of uTWEAK. uTWEAK levels were higher in patients with active LN and in Class V LN (Fig. 1B). uTWEAK levels were significantly correlated with 24 hours proteinuria, SLEDAI (Fig. 1C) and serum anti-C1q titers. An ROC curve constructed showed a good level of sensitivity and specificity (Fig. 1D)

Conclusions In our cohort of Colombian SLE patients, uTWEAK levels were 4 and 2 times higher in LN patients and ALA respectively. UTWEAK were significantly higher in active LN and were correlated with disease activity, proteinuria and anti-C1q antibodies.

\section{PREVALENCE OF ANTI-DFS70 ANTIBODIES IN A COLOMBIAN COHORT: A CASE-CONTROL STUDY}

${ }^{1} \mathrm{~S}$ Arteaga, ${ }^{2} \mathrm{~T}$ Urrego, ${ }^{3} \mathrm{LM}$ Peñaranda, ${ }^{4} \mathrm{~A}$ Londoño, ${ }^{4} \mathrm{ML}$ Cardenas, ${ }^{1} \mathrm{AL}$ Vanegas, $\mathrm{CH}$ Muñoz ${ }^{1}{ }^{1} \mathrm{M}$ Escobar, ${ }^{1} \mathrm{~W}$ Rojas, ${ }^{1,2} \mathrm{G}$ Vásquez, ${ }^{1} \mathrm{LA}$ González, ${ }^{3} \mathrm{C}$ Perez Koller, 1,2,4JA Gómez-Puerta*. 'Universidad de Antioquia, Sección de Reumatología, Medellin, Colombia; ${ }^{2}$ Universidad de Antioquia, Grupo de Inmunología Celular e Inmunogenética, Medellin, Colombia; ${ }^{3}$ Werfen, Inova Diagnostics, Bogotá, Colombia; ${ }^{4}$ Dinamica IPS, Autoinmunidad, Medellin, Colombia

\subsection{6/lupus-2017-000215.274}

Background and aims Anti-dense fine speckled 70 (anti-DFS70) antibodies were initially identified as an ANA IIF pattern from patients with interstitial cystitis; however, some recent studies showed that anti-DFS70 antibodies are common among ANA 